\title{
Intraoperative optical assessment of photodynamic therapy response of superficial oral squamous cell carcinoma
}

Daniel J. Rohrbach

Nestor Rigual

Hassan Arshad

Erin C. Tracy

Michelle T. Cooper

Gal Shafirstein

Gregory Wilding

Mihai Merzianu

Heinz Baumann

Barbara W. Henderson

Ulas Sunar 


\title{
Intraoperative optical assessment of photodynamic therapy response of superficial oral squamous cell carcinoma
}

\author{
Daniel J. Rohrbach, ${ }^{\text {a,b }}$ Nestor Rigual, ${ }^{c}$ Hassan Arshad, ${ }^{c}$ Erin C. Tracy, ${ }^{d}$ Michelle T. Cooper, ${ }^{\text {a }}$ Gal Shafirstein, ${ }^{a}$ \\ Gregory Wilding, ${ }^{\mathrm{e}}$ Mihai Merzianu, ${ }^{\mathrm{f}}$ Heinz Baumann, ${ }^{\mathrm{d}}$ Barbara W. Henderson, ${ }^{\mathrm{a}}$ and Ulas Sunar ${ }^{\mathrm{a}, \mathrm{b}, \mathrm{g}, *}$ \\ ${ }^{a}$ Roswell Park Cancer Institute, Department of Cell Stress Biology, Elm and Carlton Streets, Buffalo, New York 14263, United States \\ ${ }^{b}$ Wright State University, Department of Biomedical, Industrial and Human Factors Engineering, 207 Russ Center, Dayton, Ohio 45435, \\ United States \\ ${ }^{c}$ Roswell Park Cancer Institute, Department of Head and Neck Surgery, Elm and Carlton Streets, Buffalo, New York 14263, United States \\ ${ }^{\mathrm{d} R o s w e l l ~ P a r k ~ C a n c e r ~ I n s t i t u t e, ~ D e p a r t m e n t ~ o f ~ C e l l u l a r ~ a n d ~ M o l e c u l a r ~ B i o l o g y, ~ E l m ~ a n d ~ C a r l t o n ~ S t r e e t s, ~ B u f f a l o, ~ N e w ~ Y o r k ~ 14263, ~ U n i t e d ~ S t a t e s ~}$ \\ ${ }^{\mathrm{e}}$ Roswell Park Cancer Institute, Department of Biostatistics and Bioinformatics, Elm and Carlton Streets, Buffalo, New York 14263, United States \\ ${ }^{\mathrm{f}}$ Roswell Park Cancer Institute, Department of Pathology and Laboratory Medicine, Elm and Carlton Streets, Buffalo, New York 14263, \\ United States \\ ${ }^{9}$ State University of New York at Buffalo, Department of Biomedical Engineering, 332 Bonner Hall, Buffalo, New York 14228, United States
}

\begin{abstract}
This study investigated whether diffuse optical spectroscopy (DOS) measurements could assess clinical response to photodynamic therapy (PDT) in patients with head and neck squamous cell carcinoma (HNSCC). In addition, the correlation between parameters measured with DOS and the crosslinking of signal transducer and activator of transcription 3 (STAT3), a molecular marker for PDT-induced photoreaction, was investigated. Thirteen patients with early stage HNSCC received the photosensitizer 2-[1-hexyloxyethyl]-2-devinylpyropheophorbide-a (HPPH) and DOS measurements were performed before and after PDT in the operating room (OR). In addition, biopsies were acquired after PDT to assess the STAT3 crosslinking. Parameters measured with DOS, including blood volume fraction, blood oxygen saturation $\left(\mathrm{StO}_{2}\right), \mathrm{HPPH}$ concentration (cHPPH), HPPH fluorescence, and blood flow index (BFI), were compared to the pathologic response and the STAT3 crosslinking. The best individual predictor of pathological response was a change in $\mathrm{cHPPH}$ (sensitivity $=60 \%$, specificity $=100 \%$ ), while discrimination analysis using a two-parameter classifier (change in $\mathrm{cHPPH}$ and change in $\mathrm{StO}_{2}$ ) classified pathological response with $100 \%$ sensitivity and $100 \%$ specificity. BFI showed the best correlation with the crosslinking of STAT3. These results indicate that DOS-derived parameters can assess the clinical response in the OR, allowing for earlier reintervention if needed. ๑ 2016 Society of Photo-Optical Instrumentation Engineers (SPIE) [DOI: 10.1117/1.JBO.21.1.018002]
\end{abstract}

Keywords: diffuse optical spectroscopy; head and neck squamous cell carcinoma; photodynamic therapy; signal transducer and activator of transcription 3 crosslinking; blood flow; oxygenation; fluorescence; therapeutic response.

Paper 150609R received Sep. 11, 2015; accepted for publication Dec. 11, 2015; published online Jan. 18, 2016.

\section{Introduction}

Over the last 40 years, there has been significant effort directed toward the management of head and neck squamous cell carcinoma (HNSCC). ${ }^{1}$ The standard treatments for T1 HNSCCs are surgery and radiotherapy, ${ }^{2}$ with surgery generally giving better 5 -year survival compared to radiation. ${ }^{3,4}$ Unfortunately, surgery often requires substantial resection to ensure tumor-free margins, including the removal of functional tissue that affect speech and the swallowing function. In addition, radiotherapy can cause major adverse events such as chronic dental decay, xerostomia, and risk of mandibular osteonecrosis, all of which reduce the quality of life for the patient. ${ }^{5}$ Therefore, there is a need for a curative treatment option with no long-term toxicities.

Photodynamic therapy (PDT) is a promising treatment that has been successfully used for treating HNSCCs while preserving speech and the swallowing function. ${ }^{5-9}$ Effective PDT requires a photosensitizer (PS), oxygen, and light. ${ }^{10}$ When all three components are present in tissue, it leads to the generation

*Address all correspondence to: Ulas Sunar, E-mail: ulas.sunar@wright.edu of reactive oxygen species (ROS) that cause direct damage to tumor cells, vascular destruction, and activation of the host immune response. ${ }^{11-14}$ Unlike surgery, which has the benefit of histological samples to decide on early reintervention, assessment of PDT requires a wait-and-see approach. ${ }^{7}$ Treated lesions are monitored over the proceeding weeks and months to assess the pathological response. A major clinical need is the early assessment of response to allow for reintervention if necessary.

Quantitative optical imaging tools can play a crucial role in answering this clinical need. Diffuse optical spectroscopies (DOS) can quantify PDT-related parameters, such as tissue optical properties, blood oxygenation, and blood flow as well as PS content, making them suitable for PDT monitoring. ${ }^{15}$ DOS setups are typically portable and measurements can be performed quickly, allowing for use in the operating room (OR).

DOS measurements can be performed before, during, and after treatment. Pretreatment measurements are important to accurately characterize the lesion. For example, Amelink et al. ${ }^{16}$ performed pretreatment optical spectroscopy measurements and 
showed that SCC lesions had lower blood oxygen saturation $\left(\mathrm{StO}_{2}\right)$ and higher blood volume than noncancerous tissue. Knowledge of these parameters before treatment can provide feedback for PDT planning and response on an individual basis. Since oxygen is the critical element for PDT, hypoxic tumors are likely to respond poorly to PDT. ${ }^{17-19}$ High blood flow and blood volume may allow high PS accumulation in tumors. In addition, changes in these parameters can inform about the efficacy of the treatment. Vascular disrupting PSs like 2-[1-hexyloxyethyl]-2-devinylpyropheophorbide-a (HPPH) may induce significant changes in vascular parameters. ${ }^{20,21}$ Changes in blood flow and oxygenation can be indicative of response to PDT. ${ }^{14,21-29}$ Moreover, PS photobleaching has also been shown to be indicative of PDT response in certain cases. $^{10,29-35}$

Here we present DOS measurements acquired in the OR immediately before and after light treatment to assess clinical response. We quantified blood flow index (BFI), blood volume fraction (BVf), and $\mathrm{StO}_{2}$ as well as $\mathrm{HPPH}$ concentration (cHPPH) and fluorescence (fHPPH). Then optical measurements were compared with the pathological response and the oxidative crosslinking of the signal transducer and activator of transcription 3 (STAT3). STAT3 is a transcription factor protein expressed by essentially all cells in the body and is preferentially crosslinked in the presence of singlet oxygen. The conversion of STAT3 into a covalently linked homodimer complex has become a quantitative molecular marker for the PS-mediated photoreaction. ${ }^{36,37}$ Our study demonstrates the potential use of DOS for early assessment of the PDT response in HNSCC lesions.

\section{Materials and Methods}

\subsection{Clinical Photodynamic Therapy}

Written informed consent was obtained from all patients for all procedures. The patient treatment and measurement protocol were approved by the Roswell Park Cancer Institute Institutional Review Board. Thirteen patients with biopsy proven SCC ( $<3$-mm thick) were measured using DOS. Each patient received the PS HPPH in dextrose $5 \%$ (D5W), $4.0 \mathrm{mg} / \mathrm{m}^{2}$ infusion over $1 \mathrm{~h}$. Treatment light $\left(140 \mathrm{~J} / \mathrm{cm}^{2}, 150 \mathrm{~mW} / \mathrm{cm}^{2}\right.$, and $\left.665 \mathrm{~nm}\right)$ was delivered $\sim 24 \mathrm{~h}$ later in the OR while the patient was under monitored anesthesia. ${ }^{38}$ The treatment beam was centered on the lesion with 1 to $2 \mathrm{~cm}$ margins, with the total area of light exposure not exceeding $25 \mathrm{~cm}^{2}$. Biopsies of tumor tissue for the STAT3 crosslinking analysis were taken before and after treatment from the center of the illuminated region, immediately following the optical measurements. A schematic of the treatment protocol and DOS measurements is shown in Fig. 1.

Three months after PDT, a 3-mm punch biopsy from the treatment field was acquired for pathological response verification. Patients were examined at 3- to 6-month intervals thereafter. The responses reported here were determined from the 3-month biopsy and/or subsequent clinical observation. The biopsies were interpreted by a head and neck pathologist and were graded as shown in Table 1.

\subsection{Experimental Setup}

The instrument used in the clinic combined three diffuse optical methods: diffuse reflectance spectroscopy (DRS), diffuse fluorescence spectroscopy (DFS), and diffuse correlation spectroscopy (DCS) and has been described previously. ${ }^{20,39,40}$ Briefly, the DRS setup consisted of a tungsten-halogen broadband white light (HL-2000-FHSA, Ocean Optics, Dunedin, Florida) as the source and the master channel of a two-channel spectrometer (S2000, Ocean Optics, Dunedin, Florida) as the detector. While the wavelength range for the spectrometer was 300 to $1100 \mathrm{~nm}$, only a subset (520 to $820 \mathrm{~nm}$ ) of this range was used for DRS. The light was directed to the target with one source fiber, the diffusely reflected light was collected with one detector fiber, and the source detector (SD) separation was $1.6 \mathrm{~mm}$.

For the DFS setup, a 410-nm laser diode (Power Technologies, Little Rock, Arkansas) with an output power of $4 \mathrm{~mW}$ was used as the excitation source and the more sensitive slave channel of the spectrometer was used as the detector. A 500-nm longpass filter (450 Saffron, GAM products, Los Angeles, California) was placed in-line to reject the excitation light while passing the autofluorescence (AF) and PS fluorescence with a SD separation of $0.8 \mathrm{~mm}$. The subset of wavelengths used for DFS was 600 to $770 \mathrm{~nm}$.

The DCS setup consisted of a long coherence length, $785 \mathrm{~nm}$ laser (CrystaLaser, Reno, Nevada), four photon counting detectors (Avalanche Photodiode, Perkin Elmer, Waltham, Massachusetts) and a custom-built autocorrelator board (Correlator.com). The source was directed to the tissue by a multimode source fiber (400- $\mu \mathrm{m}$ core diameter) and collected with single-mode detector fibers (5- $\mu \mathrm{m}$ core diameter). The outputs

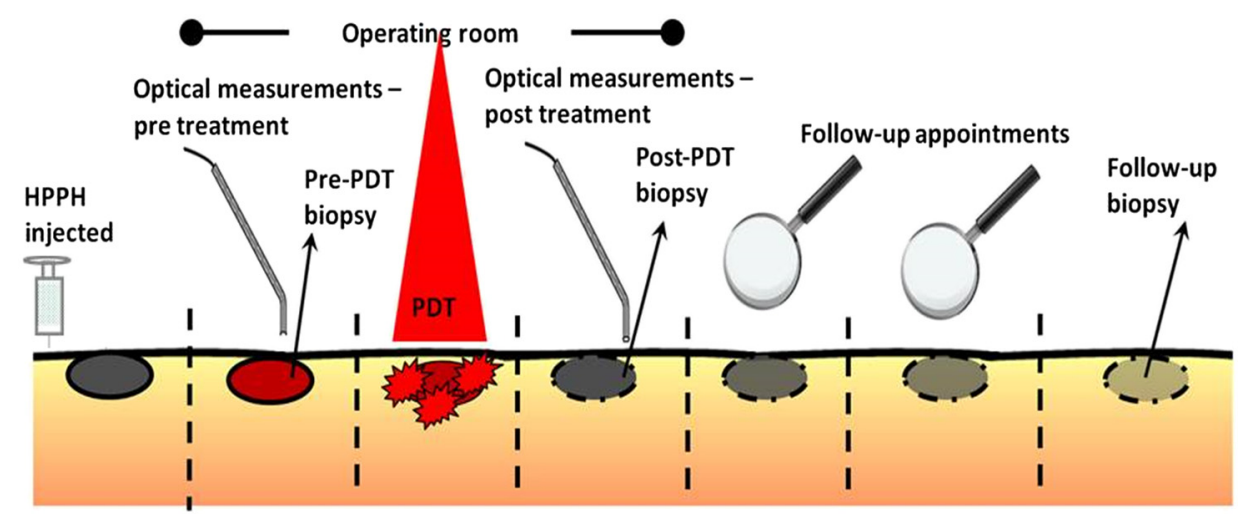

Fig. 1 Treatment plan for patients receiving PDT. The optical measurements were performed in the OR, $24 \mathrm{~h}$ after HPPH administration. Biopsies were taken after the optical measurements, both before and after treatment light illumination. Follow-up biopsies were used to assess response. 
Table 1 Response classification.

\begin{tabular}{ll} 
Grade & \multicolumn{1}{c}{ Description } \\
\hline Complete response (CR) & $\begin{array}{l}\text { Negative biopsy and/or complete } \\
\text { absence of visible lesion }\end{array}$ \\
Partial response (PR) & $\begin{array}{l}50 \% \text { or more reduction in } \\
\text { lesion volume }\end{array}$ \\
Stable disease (SD) & All responses <PR \\
Progressive disease (PD) & Increase in lesion size or stage \\
\hline
\end{tabular}

from the photodetectors were sent to the correlator board to determine the intensity autocorrelation function and photon arrival times, which were then saved by the computer. The SD separations were $0.6,1,1.2$, and $1.6 \mathrm{~mm}$, providing a measure of blood flow at various depths in the tissue.

A custom stainless steel probe (5-mm diameter) was constructed to keep all fibers in place and for ease of sterilization. All three setups were mounted on a portable cart for facilitating transport between the laboratory and the OR. Noninvasive optical measurements were performed in the OR before and after PDT while the patient was under anesthesia. Measurements consisted of the surgeon placing the probe in soft contact with the tissue surface. At least five measurements were acquired from different points on the lesion. For each probe placement, measurements were acquired sequentially with DCS first followed by the spectral measurements.

\subsection{Optical Data Analysis}

Analysis of our three DOS techniques has been discussed previously. ${ }^{20,39,40}$ Blood flow was measured using DCS. Briefly, when photons scatter from moving blood cells, the intensity of collected diffuse light fluctuates with time. The normalized electric field is extracted from the autocorrelation function and the information about the motions in deep tissue can be extracted since the electric field autocorrelation function satisfies a diffusion equation. ${ }^{41}$ An analytical solution is available for the Brownian diffusion model in reflectance measurements. The mean-square displacement $\left(\left\langle\Delta r^{2}(\tau)\right\rangle\right)$ of the red-blood cells can be modeled as $\left\langle\Delta r^{2}(\tau)\right\rangle=6 D_{\mathrm{B}} \tau$, for the case of diffusive motion; where $D_{\mathrm{B}}$ is the effective diffusion coefficient of the tissue scatterers. Empirically it has been observed that the diffusion model fits the autocorrelation curves and $\alpha D_{\mathrm{B}}$ characterizes the blood flow in deep tissue. ${ }^{42-46}$ Here, $\alpha$ represents the probability that a scattering event in tissue emanates from a moving scatterer ( $\alpha$ is generally proportional to tissue BVf). $\mathrm{BFI}$ is reported as the ratio of the measured blood flow parameter of tissue $\alpha D_{\text {Btissue }}$ to the measured blood flow parameter of a tissue-simulating phantom made by diluting $20 \%$ Intralipid (Fresenius Kabi, Grand Island, New York) in water ( $\left.D_{\text {Bintralipid }}\right)$.

Reflectance data were analyzed using an empirical look-uptable. ${ }^{40,47}$ Tissue absorption was expressed in terms of BVf, $\mathrm{StO}_{2}$, water fraction (fixed at 0.65), and $\mathrm{HPPH}$ content (cHPPH). The reduced scattering coefficient was modeled as Mie scattering. ${ }^{48,49}$ A probe-specific look-up-table was constructed by measuring 64 phantoms with known volumes of added India Ink, Intralipid, and water. ${ }^{47}$ The look-up-table incorporated probe design, construction, and instrument response into the light transport model. ${ }^{50-52}$ To extract the optical properties and physiological parameters of interest, the trustregion-reflective nonlinear fitting algorithm (lsqnonlin, MATLAB $^{\circledR}$ ) was used. ${ }^{20,39}$ The look-up-table approach was tested with tissue-simulating phantoms with known values for scattering, BVf, and cHPPH. The average error for scattering quantification was $5.8 \%$, average error for BVf was $6.8 \%$, and the average error for cHPPH was $8.1 \%$. The accuracy of extracting the $\mathrm{StO}_{2}$ was not tested with this approach.

Tissue fluorescence ( $\left.F_{\text {tissue }}\right)$ was modeled as a linear combination of fluorophores including HPPH (fHPPH) and AF, and the fluorescence data from each patient measurement were fit to the model to extract amplitudes of AF and HPPH fluorescence. $^{20,39}$ To account for the effects of tissue optical properties on the measured fluorescence, a Monte Carlo-based correction factor was determined using the optical properties at the excitation and emission wavelength extracted with DRS. ${ }^{53-55}$

\subsection{Statistical Analysis}

For binary classification, the four grades shown in Table 1 were combined into two groups: positive responders (CR) or negative responders (PR, $\mathrm{SD}$, and $\mathrm{PD})$. Receiver operating characteristic (ROC) analysis was used to assess the strength of each measured parameter to predict a particular outcome. The threshold for the sensitivity and specificity of each predictor is the maximum of the Youden index. The area under the curve (AUC) was used to assess the overall performance of each classifier. In addition, a multiple regression method was used to combine two parameters into a single classifier for ROC analysis, which was performed using MATLAB ${ }^{\circledR}$ and MedCalc software packages.

\subsection{Signal Transducer and Activator of Transcription 3 Crosslinking}

This assay quantifies the cumulative PDT-induced photoreaction. When cells or tissue are treated with PDT, immediate damages occur through ROS generated by the light-activated PS (photoreaction). ROS oxidize, among others, lipids and proteins. A preferential target for oxidation is the covalent crosslinking of latent STAT3 proportional to the absorbed PDT dose. . $^{36,37,56}$ The level of STAT3 crosslinking in the tumor was determined immediately following light treatment. Biopsy tissue was extracted by sonication in RIPA buffer. Aliquots of cleared extracts, containing $20 \mu \mathrm{g}$ proteins, were separated under denatured condition on $6 \%$ SDS polyacrylamide gels and analyzed by immunoblotting for the level of homodimeric STAT3 relative to total STAT3 as reported previously. ${ }^{36}$

\section{Results}

\subsection{Predicting Clinical Response}

ROC analysis was performed for each parameter using the binary response (responders $=1$; nonresponders $=0$ ) as the classification variable. The sensitivity, specificity [given in percent $(\%)$ ], and normalized (norm.) AUC for individual parameters are presented in Table 2.

The two best individual predictors of response were the change in $\mathrm{StO}_{2}\left(\Delta \mathrm{StO}_{2}\right)$, (sensitivity $=100 \%$, specificity $=$ $67 \%$, and $\mathrm{AUC}=0.70)$ and the change in $\mathrm{cHPPH}(\Delta \mathrm{cHPPH})$ (sensitivity $=60 \%$, specificity $=100 \%$, and AUC $=0.80$ ). As shown in Fig. 2(a) $\Delta \mathrm{StO}_{2}$ is considered fair (AUC $\geq 0.70$ ) and $\Delta$ cHPPH [Fig. 2(b)] is considered good (AUC $\geq 0.80$ ). 
Table 2 Performance of individual parameters in classifying responders versus nonresponders.

\begin{tabular}{|c|c|c|c|c|c|c|}
\hline \multirow[b]{2}{*}{ Parameter } & \multicolumn{3}{|c|}{ Pre-PDT } & \multicolumn{3}{|c|}{ Changes } \\
\hline & $\begin{array}{c}\text { Sensitivity } \\
(\%)\end{array}$ & $\begin{array}{c}\text { Specificity } \\
(\%)\end{array}$ & $\begin{array}{l}\text { AUC } \\
\text { (norm.) }\end{array}$ & $\begin{array}{c}\text { Sensitivity } \\
(\%)\end{array}$ & $\begin{array}{l}\text { Specificity } \\
(\%)\end{array}$ & $\begin{array}{c}\text { AUC } \\
\text { (norm.) }\end{array}$ \\
\hline $\mathrm{BFI}$ & 80 & 67 & 0.63 & 40 & 100 & 0.67 \\
\hline $\mathrm{StO}_{2}$ & 100 & 67 & 0.67 & 100 & 67 & 0.70 \\
\hline$B V f$ & 70 & 67 & 0.60 & 50 & 100 & 0.67 \\
\hline fHPPH & 90 & 67 & 0.67 & 100 & 33 & 0.53 \\
\hline cHPPH & 90 & 67 & 0.67 & 60 & 100 & 0.80 \\
\hline
\end{tabular}

Measured parameters include: $\mathrm{BFI}, \mathrm{StO}_{2}, \mathrm{BVf}, \mathrm{HPPH}$ content measured with fluorescence (fHPPH), and HPPH content measured with reflectance (cHPPH).
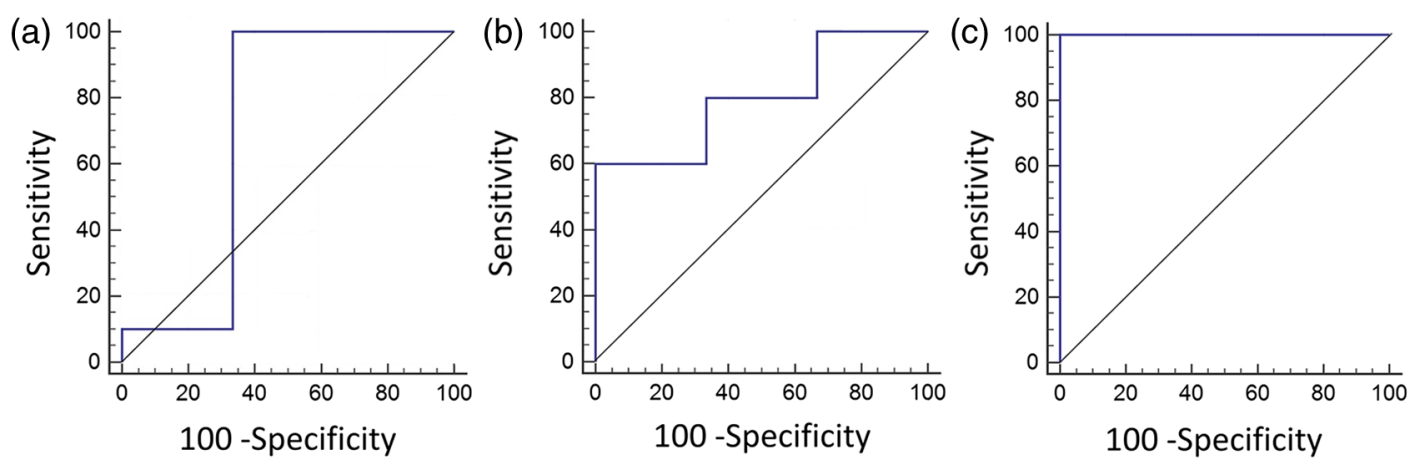

Fig. $2 \mathrm{ROC}$ curves for the best parameters for predicting response. (a) $\Delta \mathrm{StO}_{2}$ shows fair classification $(A \cup C=0.70)$ and $(b) \triangle c H P P H$ shows good classification $(A \cup C=0.80) .(c)$ The combined classifier $\left(\triangle \mathrm{StO}_{2}\right.$ and $\left.\triangle \mathrm{CHPPH}\right)$ is considered excellent for predicting pathological response (AUC $\left.=1.0\right)$.

As Table 2 summarizes, all other individual parameters were considered fair or poor.

As we and others have indicated previously, individual parameters may not be good enough for accurate discrimination of responders from nonresponders. ${ }^{20,39,57}$ Thus, the combination of multiple parameters into a single classifier was investigated with ROC analysis. A multiple regression model was used to combine several parameters into a single classifier. For each combination of two parameters, both pretreatment values and changes in these parameters were tested. The analysis showed that the best pair of classifiers was $\left[\Delta \mathrm{cHPPH}\right.$ and $\left.\Delta \mathrm{StO}_{2}\right]$. This combined classifier perfectly predicted responders and nonresponders [Fig. 2(c), sensitivity $=100 \%$, specificity $=100 \%$, and $\mathrm{AUC}=1.0]$.

\subsection{Diffuse Optical Spectroscopy Parameters Correlate with Signal Transducer and Activator of Transcription 3 Crosslinking}

As indicated in our previous studies, ${ }^{20,23,36,37,39}$ the oxidative homodimeric crosslinking of STAT3 is a molecular indicator of local photoreaction. Thus, we investigated the relationship between the PDT-related parameters obtained by noninvasive DOS measurements and the relative level of STAT3 modification determined in an extract from a single biopsy taken immediately after light treatment. There was no correlation between
BVf and STAT3 or between $\mathrm{StO}_{2}$ and STAT3. The PS-related parameters, cHPPH and $\mathrm{HHPPH}$, showed some correlation with STAT3; $r^{2}=0.46$ [Fig. 3(a)] and $r^{2}=0.41$ [Fig. 3(b)], respectively. As shown in Fig. 3(c), the parameter with the highest correlation to STAT3 crosslinking was the BFI measured before PDT $\left(r^{2}=0.61\right)$. PDT induced substantial $(>40 \%)$ changes in all DOS parameters. However, there were weak correlations between these changes and the STAT3 crosslinking. The only parameter whose change showed some correlation with STAT3 crosslinking was change in BFI $\left(r^{2}=0.42\right)$, as shown in Fig. 3(d).

For effective therapy, there needs to be PDT-induced photoreaction in the lesion. ${ }^{20,36,37,39,40}$ To investigate the ability of DOS measurements to predict whether a lesion had a photoreaction above a certain threshold, ROC analysis was performed. The classification variable was STAT3 crosslinking with a threshold set at 5\% (median of all patients). The threshold simplified the analysis as a binary classification between high crosslinking (higher than 5\%) and low crosslinking (lower than 5\%). Figure 3(e) shows the ROC analysis for the pre-PDT BFI values, which indicated the best correlation with STAT3 (86\% sensitivity, $100 \%$ specificity, and AUC $=0.93$ ). Figure 3(f) shows the ROC analysis for $\triangle \mathrm{BFI}$ which, of all the post-treatment changes, correlated best with STAT3 crosslinking (71\% sensitivity, 100\% specificity, and AUC $=0.91)$. These results indicated that optical measurements have the potential to predict the PDT-induced 

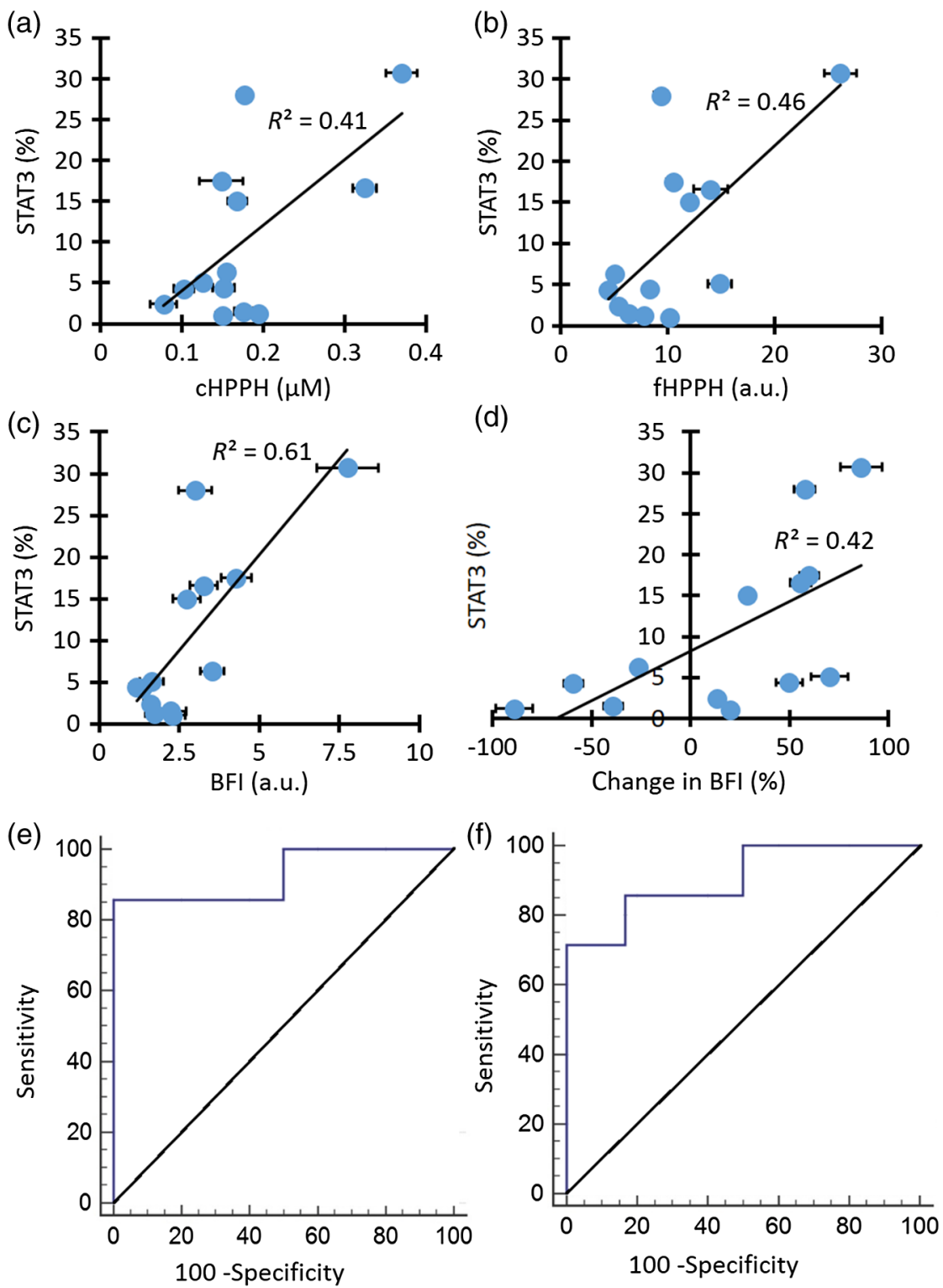

Fig. 3 Correlation of pre-PDT measurements with STAT3 crosslinking. (a) cHPPH measured with DRS, (b) fHPPH measured with DFS, (c) BFI measured with DCS, and (d) percent change in BFI. ROC analysis shows that (e) pre-PDT BFI as well as (f) $\triangle B F I$ are good parameters for classifying high and low STAT3 crosslinking. Error bars represent standard deviation of multiple $(n \geq 5)$ measurements per patient.

Table 3 Performance of individual parameters in classifying high versus low STAT3 crosslinking.

\begin{tabular}{|c|c|c|c|c|c|c|}
\hline \multirow[b]{2}{*}{ Parameter } & \multicolumn{3}{|c|}{ Pre-PDT } & \multicolumn{3}{|c|}{ Changes } \\
\hline & Sensitivity (\%) & Specificity (\%) & AUC (norm.) & Sensitivity (\%) & Specificity (\%) & AUC (norm.) \\
\hline $\mathrm{BFI}$ & 86 & 100 & 0.93 & 71 & 100 & 0.91 \\
\hline $\mathrm{StO}_{2}$ & 100 & 50 & 0.71 & 100 & 50 & 0.56 \\
\hline BVf & 29 & 50 & 0.54 & 71 & 83 & 0.74 \\
\hline $\mathrm{fHPPH}$ & 71 & 100 & 0.86 & 43 & 100 & 0.57 \\
\hline cHPPH & 71 & 67 & 0.69 & 43 & 83 & 0.55 \\
\hline
\end{tabular}

Measured parameters include: $\mathrm{BFI}, \mathrm{StO}_{2}, \mathrm{BVf}, \mathrm{HPPH}$ content measured with fluorescence (fHPPH), and HPPH content measured with reflectance (cHPPH). 
photoreaction. A summary of the ROC analysis for all parameters at discriminating between high and low STAT3 crosslinking is shown in Table 3 .

\section{Discussion}

Among pre-PDT DOS-derived parameters and changes thereof, we found that no individual parameter could determine patient response with both high sensitivity and high specificity. The best individual parameter was change in PS concentration $(\Delta \mathrm{cHPPH})$. When multiple parameters were combined into a single classifier, the best predictor for favorable response was change in PS and tissue oxygen saturation ( $\left.\left[\Delta \mathrm{cHPPH}, \Delta \mathrm{StO}_{2}\right]\right)$. This set of parameters was a perfect classifier having $100 \%$ sensitivity, $100 \%$ specificity, and an AUC of 1.0. It is important to note that our findings imply effective PDT requires the consumption of both PS and oxygen, as is expected. Pre-PDT values were also indicators of the response with a lesser power. This may indicate that, for the shallow lesions as in this study, prePDT oxygen or PS content is not the limiting factor for the lesions to respond to PDT. We should also note that there were only 13 patients investigated and only three were negative responders. More patients are needed to confirm this combined classifier, but our results show that quantification of vascular parameters and PS content have potential for early assessment of the PDT response.

It is interesting to note that while $\Delta \mathrm{cHPPH}$ was a good predictor of response $(\mathrm{AUC}=0.80), \Delta \mathrm{fHPPH}$ was not (AUC $=0.53$ ). A possible explanation is that DFS measurements were probing much more superficial tissue due to the shorter SD separation and the 410-nm excitation wavelength, where scattering and absorption parameters are high and light penetration depth is low. It is possible that the DFS measurements mainly sampled the surface directly under the fiber tip, the very superficial layer of SCCs, while the DRS measurements interrogated the lesion more thoroughly.

DOS-derived individual parameters at pre-PDT showed some level of correlation with the PDT-induced photoreaction quantified with STAT3 crosslinking analysis. Pre-PDT BFI had the highest correlation with STAT3 crosslinking $\left(r^{2}=0.61\right)$. Pre-PDT BFI parameter was also the best parameter for binary classification of high versus low photoreaction, with an AUC of 0.90. These results indicate that pre-PDT BFI measurements can potentially predict the PDT-induced photoreaction above a certain threshold within the lesion.

Changes in individual parameters generally showed worse correlation with STAT3 crosslinking than pre-PDT values. Only changes in BFI showed some correlation $\left(r^{2}=0.42\right)$ with STAT3 crosslinking and some ability to predict high versus low photoreaction. The reason for the smaller correlation between the changes in DOS parameters and STAT3 crosslinking is not well understood, but overall this may be due to variations in the tissue sampling. Since coregistering pre- and post-PDT measurements is difficult, multiple measurements were acquired from different locations on the lesion whereas STAT3 crosslinking was measured from a single biopsy. Thus, we expect averaged optical measurements would lead to more accurate result than STAT3 analysis originating from a single biopsy sample. Our PDT protocol only included lesions less than 3-mm thick, which can lead to higher sampling errors for thinner lesions. Variation in optical probe pressure can also affect the DOS-derived physiologic parameters. ${ }^{58}$
PDT causes tissue destruction based on PS activation by light under available oxygen. Since PDT efficacy is strongly dependent on light, PS, and oxygen, it is desirable to quantify these parameters. Knowledge of these parameters before PDT can be used to improve therapy planning and optimization. In this respect, light distribution and PS content are usually quantified pre-PDT time point; this is called explicit dosimetry. ${ }^{30}$ In this study, a predetermined, fixed light dose was implemented although the local light dose depends on tissue optical parameters and can vary between the lesions. Similarly, although the same amount of PS is injected for each patient, PS accumulation in each lesion can vary depending on the physiologic parameters such as lesion vascularity, drug delivery, and cellular retention. ${ }^{59,60}$ DOS techniques can provide local PDT-related parameters such as tissue optical properties, blood flow, oxygenation, and PS content. Thus, assessing these parameters locally with DOS can provide useful feedback for PDT planning for improved PDT efficacy.

PDT is a dynamic process and these physiologic parameters change continuously during PDT. These changes can provide feedback for PDT response. DOS techniques are fast and noninvasive allowing repeated measurements in the OR. The measured parameters can potentially quantify the photoreaction in the lesion and assess the response. It has been shown for some cases that PS photobleaching, blood flow, and oxygenation dynamics are indicative of PDT response. ${ }^{14,30,61}$

There is room for improvement of optical instrumentation for monitoring PDT of SCC lesions in the oral cavity. In its current form, the setup could not perform continuous measurements. In the future, a compact noncontact probe that includes the treatment fiber and allows continuous measurements could assess the dynamics of PDT-related parameters. Pre- and post-PDT time points provide limited information in terms of the dynamics of the process. We have shown recently that blood flow dynamics correlated well with the STAT3 crosslinking $\left(r^{2}=0.87\right)$ in a head and neck mouse tumor model. ${ }^{23}$ Next, since oral lesions show significant heterogeneity, optical imaging would be a better approach to demarcate the whole lesion and adjust the treatment plan according to this image guidance.

\section{Conclusion}

Successful PDT is a result of optimal distributions of light, PS, and oxygen. DOS measurements can provide PDT-related parameters such as PS content and tissue blood oxygenation. Since DOS is a noninvasive technique, it can provide multiple in vivo tissue measurements that characterize tumor properties relevant to PDT. Changes in DOS parameters can inform about the dynamic nature of PDT such as PS photobleaching and oxygen consumption. We showed that the combination of multiple parameters measured in the OR allowed better prediction of the clinical response assessed 6-month post-PDT. Weak correlation with the STAT3 biopsy analysis shows the variations in biopsy sampling, especially for very small and superficial lesions, and highlights the benefit of noninvasive sampling during DOS measurements. Ultimately, we expect the feedback provided by the in vivo metrics quantified with DOS will allow for adapting the treatment protocols when needed for improved therapeutic outcome on an individual basis and earlier reintervention if one treatment is not successful.

\section{Acknowledgments}

The authors would like to thank Ken Keymel and Brian Wrazen for their technical assistance. This research was partially supported by the NCI Grants Nos. CA55791 and P30CA16056. 


\section{References}

1. H. Quon et al., "Photodynamic therapy in the management of pre-malignant head and neck mucosal dysplasia and microinvasive carcinoma," Photodiagn. Photodyn. Ther. 8(2), 75-85 (2011).

2. T. A. Day et al., "Oral cancer treatment," Curr. Treat Options Oncol. 4(1), 27-41 (2003).

3. L. Thomas et al., "Long-term quality of life in young adults treated for oral cavity squamous cell cancer," Ann. Otol. Rhinol. Laryngol. 121(6), 395-401 (2012).

4. M. Umeda et al., "A comparison of brachytherapy and surgery for the treatment of stage I-II squamous cell carcinoma of the tongue," Int. J. Oral. Maxillofac. Surg. 34(7), 739-744 (2005).

5. M. A. Biel, "Photodynamic therapy treatment of early oral and laryngeal cancers," Photochem. Photobiol. 83(5), 1063-1068 (2007).

6. N. R. Rigual et al., "Photodynamic therapy for head and neck dysplasia and cancer," Arch. Otolaryngol. Head Neck Surg. 135(8), 784-788 (2009).

7. S. A. de Visscher et al., "mTHPC-mediated photodynamic therapy of early stage oral squamous cell carcinoma: a comparison to surgical treatment," Ann. Surg. Oncol. 20(9), 3076-3082 (2013).

8. H. Ikeda et al., "Treatment outcome of photofrin-based photodynamic therapy for T1 and T2 oral squamous cell carcinoma and dysplasia," Photodiagn. Photodyn. Ther. 10(3), 229-235 (2013).

9. M. P. von Beckerath et al., "Outcome of primary treatment of early laryngeal malignancies using photodynamic therapy," Acta Otolaryngol. 134(8), 852-858 (2014).

10. B. C. Wilson and M. S. Patterson, "The physics, biophysics and technology of photodynamic therapy," Phys. Med. Biol. 53(9), R61-R109 (2008).

11. B. W. Henderson, T. M. Busch, and J. W. Snyder, "Fluence rate as a modulator of PDT mechanisms," Lasers Surg. Med. 38(5), 489-493 (2006).

12. B. W. Henderson et al., "Choice of oxygen-conserving treatment regimen determines the inflammatory response and outcome of photodynamic therapy of tumors," Cancer Res. 64(6), 2120-2126 (2004).

13. T. M. Busch et al., "Increasing damage to tumor blood vessels during motexafin lutetium-PDT through use of low fluence rate," Radiat. Res. 174(3), 331-340 (2010)

14. G. Yu et al., "Noninvasive monitoring of murine tumor blood flow during and after photodynamic therapy provides early assessment of therapeutic efficacy," Clin. Cancer Res. 11(9), 3543-3552 (2005).

15. S. M. Gallagher-Colombo et al., "Measuring the physiologic properties of oral lesions receiving fractionated photodynamic therapy," Photochem. Photobiol. 91(5), 1210-1218 (2015).

16. A. Amelink et al., "Non-invasive measurement of the morphology and physiology of oral mucosa by use of optical spectroscopy," Oral Oncol. 44(1), 65-71 (2008).

17. M. P. Bard et al., "Measurement of hypoxia-related parameters in bronchial mucosa by use of optical spectroscopy," Am. J. Respir. Crit. Care Med. 171(10), 1178-1184 (2005).

18. B. J. Tromberg et al., "In vivo tumor oxygen tension measurements for the evaluation of the efficiency of photodynamic therapy," Photochem. Photobiol. 52(2), 375-385 (1990).

19. T. H. Foster et al., "Oxygen consumption and diffusion effects in photodynamic therapy," Radiat. Res. 126(3), 296-303 (1991).

20. U. Sunar et al., "Monitoring photobleaching and hemodynamic responses to HPPH-mediated photodynamic therapy of head and neck cancer: a case report," Opt. Express 18(14), 14969-14978 (2010).

21. J. Dong et al., "Hemodynamic monitoring of chlorin e6-mediated photodynamic therapy using diffuse optical measurements," J. Photochem. Photobiol. B 140, 163-172 (2014).

22. B. Chen et al., "Blood flow dynamics after photodynamic therapy with verteporfin in the RIF-1 tumor," Radiat. Res. 160(4), 452-459 (2003).

23. D. J. Rohrbach et al., "Blood flow dynamics during local photoreaction in a head and neck tumor model," Front. Phys. 3, 13 (2015).

24. H. Li et al., "Feasibility of interstitial Doppler optical coherence tomography for in vivo detection of microvascular changes during photodynamic therapy," Lasers Surg. Med. 38(8), 754-761 (2006).

25. H. W. Wang et al., "Treatment-induced changes in tumor oxygenation predict photodynamic therapy outcome," Cancer Res. 64(20), 75537561 (2004).
26. T. H. Pham et al., "Monitoring tumor response during photodynamic therapy using near-infrared photon-migration spectroscopy," Photochem. Photobiol. 73(6), 669-677 (2001).

27. J. H. Woodhams et al., "Correlation of real-time haemoglobin oxygen saturation monitoring during photodynamic therapy with microvascular effects and tissue necrosis in normal rat liver," Br. J. Cancer 91(4), 788-794 (2004).

28. A. Amelink et al., "Monitoring PDT by means of superficial reflectance spectroscopy," J. Photochem. Photobiol. B 79(3), 243-251 (2005).

29. B. Karakullukcu et al., "Integration of fluorescence differential pathlength spectroscopy to photodynamic therapy of the head and neck tumors is useful in monitoring clinical outcome," Front. Phys. 3, 20 (2015).

30. B. C. Wilson, M. S. Patterson, and L. Lilge, "Implicit and explicit dosimetry in photodynamic therapy: a new paradigm," Lasers Med. Sci. 12(3), 182-199 (1997).

31. I. A. Boere et al., "Monitoring in situ dosimetry and protoporphyrin IX fluorescence photobleaching in the normal rat esophagus during 5-aminolevulinic acid photodynamic therapy," Photochem. Photobiol. 78(3), 271-277 (2003).

32. I. Georgakoudi and T. H. Foster, "Singlet oxygen- versus nonsinglet oxygen-mediated mechanisms of sensitizer photobleaching and their effects on photodynamic dosimetry," Photochem. Photobiol. 67(6), 612-625 (1998).

33. D. J. Robinson et al., "Fluorescence photobleaching of ALA-induced protoporphyrin IX during photodynamic therapy of normal hairless mouse skin: the effect of light dose and irradiance and the resulting biological effect," Photochem. Photobiol. 67(1), 140-149 (1998).

34. C. Sheng et al., "Photobleaching-based dosimetry predicts deposited dose in ALA-PpIX PDT of rodent esophagus," Photochem. Photobiol. 83(3), 738-748 (2007).

35. U. Sunar, "Monitoring photodynamic therapy of head and neck malignancies with optical spectroscopies," World J. Clin. Cases 1(3), 96-105 (2013).

36. B. W. Henderson et al., "Cross-linking of signal transducer and activator of transcription 3-a molecular marker for the photodynamic reaction in cells and tumors," Clin. Cancer Res. 13(11), 3156-3163 (2007).

37. W. Liu, A. R. Oseroff, and H. Baumann, "Photodynamic therapy causes cross-linking of signal transducer and activator of transcription proteins and attenuation of interleukin- 6 cytokine responsiveness in epithelial cells," Cancer Res. 64(18), 6579-6587 (2004).

38. N. Rigual et al., "Photodynamic therapy with 3-(1'-hexyloxyethyl)pyropheophorbide a for cancer of the oral cavity," Clin. Cancer Res. 19(23), 6605-6613 (2013).

39. D. J. Rohrbach et al., "Interlesion differences in the local photodynamic therapy response of oral cavity lesions assessed by diffuse optical spectroscopies," Biomed. Opt. Express 3(9), 2142-2153 (2012).

40. D. J. Rohrbach et al., "Monitoring PDT response of head and neck lesions with diffuse optical spectroscopies," Proc. SPIE 8568, 856814 (2013).

41. D. A. Boas, L. E. Campbell, and A. G. Yodh, "Scattering and imaging with diffusing temporal field correlations," Phys. Rev. Lett. 75(9), 1855-1858 (1995).

42. R. C. Mesquita et al., "Direct measurement of tissue blood flow and metabolism with diffuse optics," Philos. Trans. A Math. Phys. Eng. Sci. 369(1955), 4390-4406 (2011).

43. C. Cheung et al., "In vivo cerebrovascular measurement combining diffuse near-infrared absorption and correlation spectroscopies," Phys. Med. Biol. 46(8), 2053-2065 (2001).

44. U. Sunar et al., "Hemodynamic responses to antivascular therapy and ionizing radiation assessed by diffuse optical spectroscopies," Opt. Express 15, 15507-15516 (2007).

45. U. Sunar et al., "Noninvasive diffuse optical measurement of blood flow and blood oxygenation for monitoring radiation therapy in patients with head and neck tumors: a pilot study," J. Biomed. Opt. 11(6), 064021 (2006).

46. S. A. Carp et al., "Validation of diffuse correlation spectroscopy measurements of rodent cerebral blood flow with simultaneous arterial spin labeling MRI; towards MRI-optical continuous cerebral metabolic monitoring," Biomed. Opt. Express 1(2), 553-565 (2010).

47. D. J. Rohrbach et al., "A probe specific empirical light transport model for improved quantification of optical parameters for accurate PDT dosimetry," Proc. SPIE 8568, 856815 (2013). 
48. J. R. Mourant et al., "Predictions and measurements of scattering and absorption over broad wavelength ranges in tissue phantoms," Appl. Opt. 36(4), 949-957 (1997).

49. S. L. Jacques, "Origins of tissue optical properties in the UVA, visible, and NIR regions," OSA TOPS Adv. Opt. Imaging Photon Migr. 2, 364 369 (1996).

50. B. S. Nichols, N. Rajaram, and J. W. Tunnell, "Performance of a lookup table-based approach for measuring tissue optical properties with diffuse optical spectroscopy," J. Biomed. Opt. 17(5), 057001 (2012).

51. N. Rajaram, T. H. Nguyen, and J. W. Tunnell, "Lookup table-based inverse model for determining optical properties of turbid media," J. Biomed. Opt. 13(5), 050501 (2008).

52. P. R. Bargo et al., "In vivo determination of optical properties of normal and tumor tissue with white light reflectance and an empirical light transport model during endoscopy," J. Biomed. Opt. 10(3), 034018 (2005).

53. Q. Liu, C. Zhu, and N. Ramanujam, "Experimental validation of Monte Carlo modeling of fluorescence in tissues in the UV-visible spectrum," J. Biomed. Opt. 8(2), 223-236 (2003).

54. J. Swartling et al., "Accelerated Monte Carlo models to simulate fluorescence spectra from layered tissues," J. Opt. Soc. Am. A. Opt. Image. Sci. Vis. 20(4), 714-727 (2003).

55. C. Liu et al., "Experimental validation of an inverse fluorescence Monte Carlo model to extract concentrations of metabolically relevant fluorophores from turbid phantoms and a murine tumor model," J. Biomed. Opt. 17(7), 078003 (2012).

56. A. Srivatsan et al., "In vitro cellular uptake and dimerization of signal transducer and activator of transcription-3 (STAT3) identify the photosensitizing and imaging-potential of isomeric photosensitizers derived from chlorophyll-a and bacteriochlorophyll-a," J. Med. Chem. 54(19), 6859-6873 (2011)

57. A. Amelink et al., "Non-invasive measurement of the microvascular properties of non-dysplastic and dysplastic oral leukoplakias by use of optical spectroscopy," Oral Oncol. 47(12), 1165-1170 (2011).

58. L. Lim et al., "Probe pressure effects on human skin diffuse reflectance and fluorescence spectroscopy measurements," J. Biomed. Opt. 16(1), 011012 (2011).

59. A. Srivatsan et al., "Effect of chirality on cellular uptake, imaging and photodynamic therapy of photosensitizers derived from chlorophyll-a," Bioorg. Med. Chem. 23(13), 3603-3617 (2015).

60. E. C. Tracy et al., "Cell-type selective phototoxicity achieved with chlorophyll-a derived photosensitizers in a co-culture system of primary human tumor and normal lung cells," Photochem. Photobiol. 87(6), 1405-1418 (2011).

61. G. Yu, "Near-infrared diffuse correlation spectroscopy in cancer diagnosis and therapy monitoring," J. Biomed. Opt. 17(1), 010901 (2012).

Biographies for the authors are not available. 